\title{
Neural Correlates of Response Inhibition and Cigarette Smoking in Late Adolescence
}

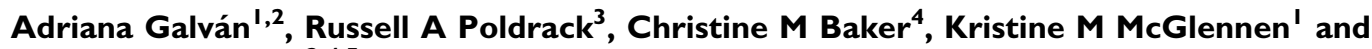 \\ Edythe D London ${ }^{*, 2,4,5}$
}

'Department of Psychology, University of California, Los Angeles, CA, USA; ${ }^{2}$ Brain Research Institute, University of California, Los Angeles, CA, USA; ${ }^{3}$ maging Research Center, University of Texas, Austin, TX, USA; ${ }^{4}$ Department of Psychiatry and Biobehavioral Sciences, Semel Institute for Neuroscience and Human Behavior, David Geffen School of Medicine, University of California, Los Angeles, CA, USA; ${ }^{5}$ Department of Molecular and Medical Pharmacology, University of California, Los Angeles, CA, USA

Smoking is usually initiated in adolescence, and is the leading preventable cause of death in the United States. Little is known, however, about the links between smoking and neurobiological function in adolescent smokers. This study aimed to probe prefrontal cortical function in late adolescent smokers, using a response inhibition task, and to assess possible relationships between inhibition-related brain activity, clinical features of smoking behavior, and exposure to cigarette smoking. Participants in this study were otherwise healthy late adolescent smokers ( $15-2 \mid$ years of age; $n=25)$, who reported daily smoking for at least the 6 months before testing, and age- and education-matched nonsmokers ( $|6-2|$ years of age; $n=25)$, who each reported smoking fewer than five cigarettes in their lifetimes. The subjects performed the Stop-signal Task, while undergoing functional magnetic resonance imaging. There were no significant group differences in prefrontal cortical activity during response inhibition, but the Heaviness of Smoking Index, a measure of smoking behavior and dependence, was negatively related to neural function in cortical regions of the smokers. These findings suggest that smoking can modulate prefrontal cortical function. Given the late development of the prefrontal cortex, which continues through adolescence, it is possible that smoking may influence the trajectory of brain development during this critical developmental period. Neuropsychopharmacology (20II) 36, 970-978; doi:I 0.1038/npp.20 I0.235; published online 26 January 201 I

Keywords: adolescence; smoking; $\mathrm{PMRl}$; inhibitory control; brain development

\section{INTRODUCTION}

Tobacco smoking is the leading preventable cause of disease and death in the United States (Center for Disease Control and Prevention, 2008). Approximately $80 \%$ of adult smokers have progressed to nicotine dependence by age of 18 years (US DHHS), and people who do not start smoking as teenagers are unlikely ever to do so (Sussman, 2002). Nicotine exposure during development has been linked to subsequent deficits in attention and memory, and in underlying neural circuitry (Jacobsen et al, 2007), but there is more to learn about the interactions of smoking with the adolescent brain. Particularly relevant is the fact that the prefrontal cortex (PFC) continues to develop structurally (Giedd and Rapoport, 2010) and functionally (Galván et al, 2006; Somerville and Casey, 2010) into adulthood. This

*Correspondence: Dr ED London, Department of Psychiatry and Biobehavioral Sciences, Semel Institute for Neuroscience and Human Behavior, David Geffen School of Medicine, University of California, Los Angeles 760 Westwood Plaza, Los Angeles, CA 90024-1759, USA, Tel: + | 310825 0606, Fax: + | 3108250812 ,

E-mail: elondon@mednet.ucla.edu

Received I September 20 10; revised 8 November 2010; accepted 29 November 2010 protracted development has been implicated as a cause of maladaptive decision-making associated with immature cognitive control during adolescence (Ernst et al, 2009b; Somerville and Casey, 2010).

Developmental plasticity renders the brain vulnerable to the effects of nicotine and smoking (Dwyer et al, 2009; Mathers et al, 2006). In rodents, frontostriatal circuitry is especially susceptible to nicotine exposure during adolescence (Schochet et al, 2005), with subsequent cognitive deficits in adulthood (Counett et al, 2009; Fountain et al, 2008). It has been proposed that neurotoxic effects during adolescence, when neural circuitry that subserves inhibitory control undergoes significant development, explain why early initiation of cigarette smoking leads to higher levels of nicotine dependence (Mathers et al, 2006).

Using functional magnetic resonance imaging (fMRI) and the Stop-signal Task (SST) (Logan, 1994) as a probe to evaluate PFC function, we assessed the neural correlates of response inhibition in late adolescent smokers and nonsmokers. We selected the SST because performance on the task has been shown to correlate reliably with prefrontal cortical activation (Aron and Poldrack, 2006; Chikazoe et al, 2009; Duann et al, 2009; Li et al, 2006). Young adult smokers exhibit a greater percentage of errors than 
nonsmokers, but stopping ability does not differ with smoking status (Monterosso et al, 2005). We reasoned, however, that younger participants, undergoing PFC development, might exhibit smoking-related deficits in taskrelated activity.

Successful response inhibition is associated with activation in the right inferior frontal gyrus (IFG) in nonsmokers (Aron and Poldrack, 2006), a region that undergoes significant neurodevelopmental change during adolescence (eg, Durston et al, 2006; Giedd and Rapoport, 2010; Velanova et al, 2008). If smoking were to influence the trajectory of development, we would expect smokers to rely less on this region and more on a distributed network, similar to findings previously shown using response inhibition tasks in youth at high risk for substance use disorders (McNamee et al, 2008; Schweinsburg et al, 2004). In these studies, there was a negative association between response inhibition and prefrontal activation in youth at-risk for developing substance use disorders. Further, our hypothesis is based on evidence that the immature or addicted brain has inefficient function and shows a more diffuse pattern of activation than the healthy, mature brain under identical cognitive demands (Brown et al, 2005; Durston et al, 2006). We reasoned further that task-related activity in PFC regions, critical for response inhibition, would be negatively associated with smoking behavior.

\section{SUBJECTS AND METHODS}

\section{Participants}

A total of 25 English-speaking, right-handed nonsmokers (mean age: $19.3 \pm 1.32,10$ female), and 25 daily smokers (mean age: $19 \pm 1.57,11$ female) were recruited through local newspaper and Internet advertisements. In keeping with the definition of adolescence as a gradual period of transition between childhood and adulthood (Dorn et al, 2006; Galván, 2010), we consider the participants as late adolescents. After receiving an explanation of the study, participants $\geqslant 18$ years gave written informed consent, as required by the UCLA Institutional Review Board, others gave assent, their parents giving consent.

Participants were classified as nonsmokers $(<5$ cigarettes in lifetime) or smokers (daily smoking $\geqslant 6$ months). Additional requirements for nonsmokers were carbon monoxide (CO) concentrations below 5 p.p.m. in expired air (Smokelyzer, Bedfont Scientific, Kent, UK) and urinary cotinine values below the threshold of determination (NicAlert test strips, Nymox Pharmaceutical, Hasbrouck Heights, NJ). For the $25 \%$ of nonsmokers who reported having smoked a cigarette in their lifetime, the mean number of months since last cigarette was 40 months. For smokers, the requirements were $\geqslant 6$ p.p.m. CO in expired air and/or urinary cotinine $\geqslant 200 \mathrm{ng} / \mathrm{ml}$. No participant reported a medical or neurological disorder. The Structured Clinical Interview for DSM-IV was used to exclude participants meeting diagnosis for any Axis I psychiatric disorder, including current drug abuse or dependence (except nicotine for the smokers). Abstinence from substance use (except nicotine for smokers) was confirmed by urine drug screening on test days.
To assess nicotine dependence, we administered the Cigarette Dependence Scale (CDS-12) (Etter et al, 2003), which is similar to the Fagerström Test for Nicotine Dependence (FTND) (Fagerström and Schneider, 1989), but reportedly more predictive of nicotine craving and withdrawal (Etter, 2008), as well as smoking abstinence (Courvoiser and Etter, 2010) than the FTND. We also calculated the Heaviness of Smoking Index (HSI), an index of smoking behavior and dependence that reliably predicts success in maintaining abstinence after smoking cessation (Borland et al, 2010). The HSI is computed by recoding and summing the responses to two questions asked on the CDS12: the number of cigarettes smoked per day and the time to the first cigarette of the day (Borland et al, 2010). HSI values for the current sample (see Table 1) were used to test associations between smoking behavior/dependence and brain activity.

Participants did not smoke before scanning (duration of smoking abstinence range: 30-1050 min). Nicotine withdrawal can influence cognitive performance in smokers

Table I Characteristics of Research Participants

\begin{tabular}{lcc}
\hline Group & $\begin{array}{c}\text { Nonsmokers } \\
(\mathbf{n}=\mathbf{2 5})\end{array}$ & $\begin{array}{c}\text { Smokers } \\
(\mathbf{n}=\mathbf{2 5})\end{array}$ \\
\hline Sex $(\mathrm{M} / \mathrm{F})$ & $14 / 1 \mid$ & $15 / 10$ \\
Age (years) & $19(\mid .32)$ & $19.32(\mid .57)$ \\
& $($ range: $|6-2|)$ & $($ range: $|5-2|)$
\end{tabular}

\begin{tabular}{|c|c|c|}
\hline Ethnicity & & \\
\hline White Caucasian & $12 \%(n=3)$ & $20 \%(n=5)$ \\
\hline African American & $16 \%(n=4)$ & $4 \%(n=1)$ \\
\hline Hispanic & $4 \%(n=1)$ & $20 \%(n=5)$ \\
\hline Asian & $36 \%(n=9)$ & $32 \%(n=8)$ \\
\hline Native American & $4 \%(n=1)$ & $4 \%(n=1)$ \\
\hline Other & $28 \%(n=7)$ & $20 \%(n=5)$ \\
\hline Education (years) & $13.44(1.35)$ & $13.54(1.35)$ \\
\hline $\begin{array}{l}\text { Age of onset (years) weekly } \\
\text { smoking }\end{array}$ & $N / A$ & $\begin{array}{c}17(1.8) \\
\text { (range: } 13-20)\end{array}$ \\
\hline Smoking duration (months) & N/A & $\begin{array}{c}2.6(1.64) \\
\text { (range: } 5 \text { months }-8 \text { years.) }\end{array}$ \\
\hline Cigarettes/day & N/A & $\begin{array}{c}6.72(2.82) \\
\text { (range: } 3-15)\end{array}$ \\
\hline Smoking exposure (months) & $\mathrm{N} / \mathrm{A}$ & $\begin{array}{c}1.06(1.24) \\
\text { (range: } 1.4 \text { months-6 years) }\end{array}$ \\
\hline Cigarette Dependence Scale & N/A & $\begin{array}{c}36(6.99) \\
\text { (range: } 21-48)\end{array}$ \\
\hline $\begin{array}{l}\text { Time to first cigarette of the day } \\
\text { (minutes after waking) }\end{array}$ & $N / A$ & $\begin{array}{l}65.9 \mid(58.52) \\
\text { (range: } 6-180)\end{array}$ \\
\hline Heaviness of Smoking Index & N/A & $\begin{array}{l}1.21(1.04) \\
\text { (range: } 0-3)\end{array}$ \\
\hline Marijuana (joints/week) & $0.12(0.33)$ & $1.12(1.78)$ \\
\hline Alcohol (drinks/week) & 1.58 (3.02) & $5.27(5.72)^{*}$ \\
\hline
\end{tabular}

Abbreviations: F, female; $M$, male.

Data are presented as mean values (SD in parentheses).

There were no significant differences between groups except on alcohol consumption (number of drinks consumed per week) $* p<0.00$ I by

Student's t-test. 
(Azizian et al, 2009; Mendrek et al, 2006; Xu et al, 2005, 2007), and to minimize such effects we did not impose abstinence of more than 30 min nor did we instruct participants to smoke up to $30 \mathrm{~min}$ before the scan if they ordinarily would not have smoked.

Immediately before scanning, cigarette craving was assessed using the Urge to Smoke Scale (Jarvik et al, 2000). The Minnesota Nicotine Withdrawal Questionnaire (Hughes and Hatsukami, 2003) was administered to evaluate nicotine withdrawal over the week before scanning.

\section{Stop-Signal Task}

On each trial of the SST, a left- or right-pointing arrow appeared on the screen and was displayed until the participant responded or up to $1 \mathrm{~s}$. Participants were instructed to respond as quickly as possible with a left or right key press. On $25 \%$ of trials, a stop signal (auditory tone, $900 \mathrm{~Hz}, 500 \mathrm{msec}$ ) was sounded, signaling the participant to withhold his/her response. After the response or $1 \mathrm{~s}$ (if inhibition was successful), the stimulus disappeared and was followed by a jittered delay $(0.5-4 \mathrm{~s}$, mean $=1 \mathrm{~s})$. The interval between the stimulus and the stop signal (ie, stop signal delay, SSD) was varied with each participant's performance. The SSD was increased by $50 \mathrm{msec}$ after successful inhibition, and decreased by $50 \mathrm{msec}$ after inhibition failure. This procedure ensured that subjects successfully inhibited their responses on approximately $50 \%$ of inhibition trials so that difficulty level was individualized across subjects, and both behavioral performance and number of successful stop trials were equated across participants.

The initial SSD was selected from one of two interleaved algorithms (staircases), each starting with SSD values of 200 and $320 \mathrm{~ms}$ and then increased or decreased according to the participant's performance. In Run 2, the last SSD of each staircase on the first run was used as the starting value of each staircase. There were 96 Go and 32 Stop trials per run, with equal numbers of leftward and rightward-pointing arrows. Participants performed two runs of the task (256 trials total).

\section{Procedure}

All participants received SST training immediately before scanning. They were told that correctly responding and inhibiting were equally important and that it would not always be possible to inhibit a response. Stop tones were presented through headphones, and responses were registered with an MR-compatible button box.

\section{Behavioral Analysis}

Average SSD was computed for each participant from the values of the two staircases after convergence on 50\% inhibition, and SSRT was averaged across both runs. All participants responded on $>75 \%$ of Go trials, with $>90 \%$ accuracy on Go trials and $40-60 \%$ successful inhibition (Table 2).
Table 2 Stop-Signal Task Behavioral Data

\begin{tabular}{lcc}
\hline Group & $\begin{array}{c}\text { Nonsmokers } \\
(\mathbf{n}=\mathbf{2 5})\end{array}$ & $\begin{array}{c}\text { Smokers } \\
(\mathbf{n}=\mathbf{2 5})\end{array}$ \\
\hline Median correct Go RT (ms) & $490.87(105.34)$ & $506.36(\mid 14.35)$ \\
Go discrimination errors & $1.02(1.25)$ & $1.24(1.63)$ \\
Mean Stop Signal Delay (ms) & $301.59(|| 4.9)$ & $322.93(|28.0|)$ \\
Percentage inhibition & $51.55(7.28)$ & $54.37(7.28)$ \\
Stop Signal Reaction Time (ms) & $189.28(56.19)$ & $177.88(53.57)$ \\
\hline
\end{tabular}

Data are presented as means (SD in parentheses). No significant differences were noted between groups. Go RT is speed of responding on Go trials (without errors); Go discrimination errors is the number of errors in direction of response on Go trials; Stop Signal Delay (SSD) refers to the average Stop-signal delay, compared from two staircases (see Subjects and Methods), and is the point at which $P$ (inhibit) $\simeq 50 \%$; Stop Signal Reaction Time was computed for each subject as Go RT-SSD.

\section{MRI Data Acquisition}

A 3T Siemens Trio MRI scanner was used. For each run, 182 functional $\mathrm{T}^{*}$-weighted echoplanar images (EPI) were acquired (slice thickness, $4 \mathrm{~mm} ; 34$ slices; TR, $2 \mathrm{~s}$; TE, $30 \mathrm{~ms}$; flip angle, $90^{\circ}$; matrix, $64 \times 64$; FOV, $200 \mathrm{~mm}$; voxel size, $3 \times 3 \times 4 \mathrm{~mm}^{3}$ ). Two volumes, collected at the beginning of each run to allow for T1 equilibrium effects, were discarded. A T2-weighted, matched-bandwidth (MBW), high-resolution, anatomical scan and magnetizationprepared rapid-acquisition gradient echo (MPRAGE) were acquired for each subject for registration (TR, 2.3; TE, 2.1; FOV, 256; matrix, $192 \times 192$; sagittal plane; slice thickness, $1 \mathrm{~mm}$; 160 slices). The orientation for MBW and EPI scans was oblique axial to maximize brain coverage. Matlab and Psychtoolbox (http://www.psychtoolbox.org) on an Apple Powerbook (Apple Computers, Cupertino, CA) were used for stimulus presentation and timing.

\section{Imaging Preprocessing and Registration}

Initial analysis was performed using FSL (http://www.fmrib. ox.ac.uk/fsl). Image time-courses were realigned to compensate for small head movements (Jenkinson et al, 2002). All data reported are from scans that exhibited $\leqslant 2 \mathrm{~mm}$ in translational movement. The data were smoothed using a 5-mm FWHM Gaussian kernel, and filtered in the temporal domain using a nonlinear high-pass filter (60-s cutoff). EPI images were first registered to the MBW, then to the MPRAGE, and finally into standard MNI space for group comparisons and correlation analyses.

\section{Statistical Analysis}

Demographics and task-related variables were compared between groups using two-sample Student's $t$-tests.

\section{Functional Neuroimaging Data}

FSL was used for fMRI analyses, which were restricted to the PFC a priori using a mask from the Harvard Oxford Atlas in FSL. Go, StopInhibit (Successful Stopping), StopRespond (Unsuccessful Stopping), and nuisance events (missing 
responses or errors on Go trials) were modeled after convolution with a canonical hemodynamic response function (HRF). Events ( $1 \mathrm{~s}$ ) were modeled at the time of stimulus onset. Temporal derivatives were included as covariates of no interest to improve statistical sensitivity. Null events, consisting of the jittered inter-trial interval when the screen was blank, were not explicitly modeled and therefore constituted an implicit baseline.

For each participant, and each scan, two contrasts, indexing successful response inhibition, were computed: Successful Stopping $>$ Go and Successful Stopping $>$ Unsuccessful Stopping. To determine whether possible associations with smoking variables were specific to response inhibition and did not generalize to activation related to the Go process, we also computed a Go $>$ Successful Stop contrast and Go $>$ Baseline contrast. To isolate activation related to the Go and Successful Stop processes, we computed Go $>$ Baseline and Successful Stop $>$ Baseline contrasts, respectively. Regressors of interest were created by convolving a delta function representing trial onset times with a canonical (double-gamma) HRF. A second-level, fixed-effects, voxel-wise analysis combined runs for each participant, and a one-sample $t$-test was performed at each voxel for each contrast. $Z$ statistic images were conservatively thresholded using clusters determined by $Z>2.3$ and a corrected cluster significance threshold of $p<0.05$ using the theory of Gaussian Random Fields (Beckmann and Smith, 2004). A third-level analysis was performed to compare groups using the FMRIB Local Analysis of Mixed Effects module in FSL (Beckmann and Smith, 2004).

To examine correlations of indices of smoking behavior/ dependence (HSI), craving, and exposure (pack years) with neural activity, variables of interest were modeled as explanatory variables on the contrast maps in smokers only. In these regression analyses, the outlier rejection tool in FSL was used, which automatically detects outlier data points (Woolrich, 2008). Outliers are then automatically de-weighted in the multi-subject statistics. For visualization, statistical maps were projected onto an average brain of the participants. Anatomical localization within each cluster was obtained by searching within maximum likelihood regions from the FSL Harvard-Oxford probabilistic atlas to obtain the maximum $Z$ statistic and MNI coordinates within each anatomical region. All fMRI data shown were clustercorrected at $Z=2.3, p<0.05$ and controlled for multiple comparisons using FDR in FSL.

Age of smoking initiation and current alcohol use were used as covariates in each contrast. Because alcohol use was tightly linked with smoking in our sample, each of the two variables was subjected to between-group and correlation analyses. These analyses revealed no significant group differences in correlations between these variables and brain activation. In smokers, age of initiation and alcohol use were not correlated with brain activity.

\section{RESULTS}

The groups did not differ significantly in age, education, ethnicity, or number of marijuana joints smoked per week; but did differ significantly on number of alcoholic drinks per week (Table 1) (also see Table 1 for age of initiating smoking, daily cigarette consumption, length of smoking history, lifetime exposure to smoking, mean dependence scores, and time from waking to smoking the first cigarette of the day). To determine whether age was associated with smoking behavior and/or exposure, bivariate correlation analyses between age and indices of cigarette dependence and behavior were performed. There were no significant correlations between the variables examined and age (HSI $(r=0.01, p=0.47)$ and pack years $(r=0.30$, $p=0.14))$.

Immediately before scanning, cigarette craving was scored as 3.24 on average (range: 1.2-6.3) on a scale of 1-7 (UTS). Although 14 of the subjects had smoked within $2 \mathrm{~h}$ of scanning, 11 of them had not smoked for at least $2 \mathrm{~h}$. Still, retrospective reports of nicotine withdrawal over the 7 days before testing (first nine items of the MNWS) indicated that the young smokers tested here generally did not suffer high levels of withdrawal. With a possible score of 30 , the mean score was 10.1 , with only 2 of the 25 participants reporting scores of 20 or above.

\section{Behavioral Results}

There were no significant group differences in task performance (Table 2). Inhibition success rate did not differ significantly from $50 \%$, with few discrimination errors on Go trials; and values of the SSRT were in the range reported for healthy adults (Jenkinson et al, 2002) in both groups. Median RT on Go trials was not correlated with SSRT, consistent with the assumptions underlying the Stop-signal race model (Logan, 1994).

\section{fMRI Results}

Successful stopping. Successful response inhibition (Successful Stop $>$ Baseline, Successful Stop $>$ Go, and Successful Stop $>$ Unsuccessful Stop contrasts) activated a broad neural network, consistent with previous reports (Aron and Poldrack, 2006; Congdon et al, 2010) with activation in bilateral IFG, cingulate cortex, supplementary motor area, striatum, thalamus, central opercular cortex, insular cortex, superior temporal gyrus, planum temporale, middle temporal gyrus, supramarginal gyrus, and occipital pole in both groups (Figure 1; Supplementary Table 1). There were no significant group or gender differences.

Go process. The Go process (Go $>$ Baseline contrast) activated the supplementary motor cortex, precentral gyrus, and cingulate gyrus (data not shown). There were no significant group differences, and this contrast will not be discussed further.

Relationship between neural activation and response inhibition (SSRT). Across all subjects, SSRT during successful inhibition was significantly negatively correlated with activation in the IFG, insula, cingulate, orbitofrontal cortex, and temporal gyrus. Individuals with greater stopping capacity (smaller SSRT) showed greater recruitment in these regions (Figure 2; Supplementary Table 2). There were no positive correlations between neural activation and SSRT, and no group differences in correlation between activation and SSRT. 


\section{Smoking Behavior and Neural Activation During Response Inhibition}

The HSI was negatively correlated with left and right MFG $(x=-34, y=36, z=38 ; x=30, y=36, z=44)$, cingulate gyrus $(x=0, y=14, z=28)$, supplementary motor cortex $(x=0, y=-2, z=62)$, orbitofrontal cortex $(x=-26, y=56$, $z=2)$, and right superior frontal gyrus $(x=24, y=-2$,

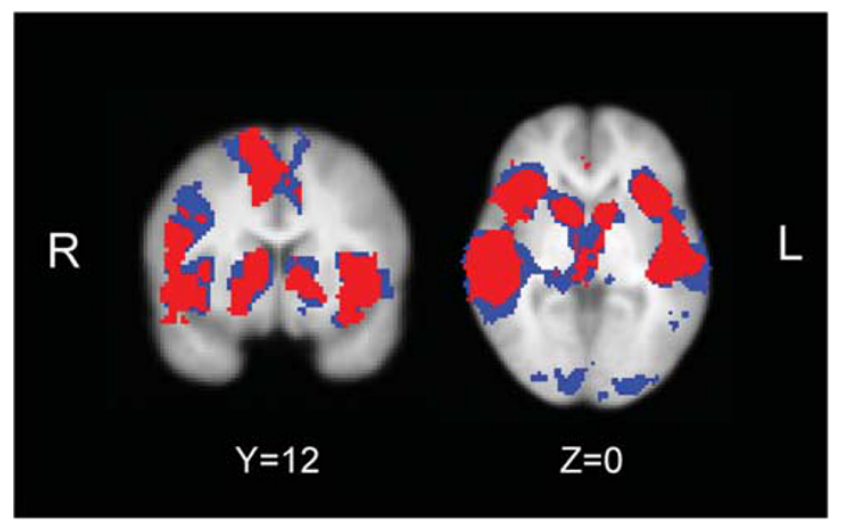

Figure I Neural activation during successful inhibition. Direct group comparisons revealed no significant differences. For illustration only, conjunction group maps of all three contrasts for nonsmokers (blue) and smokers (red) are overlaid onto the averaged normalized brain.

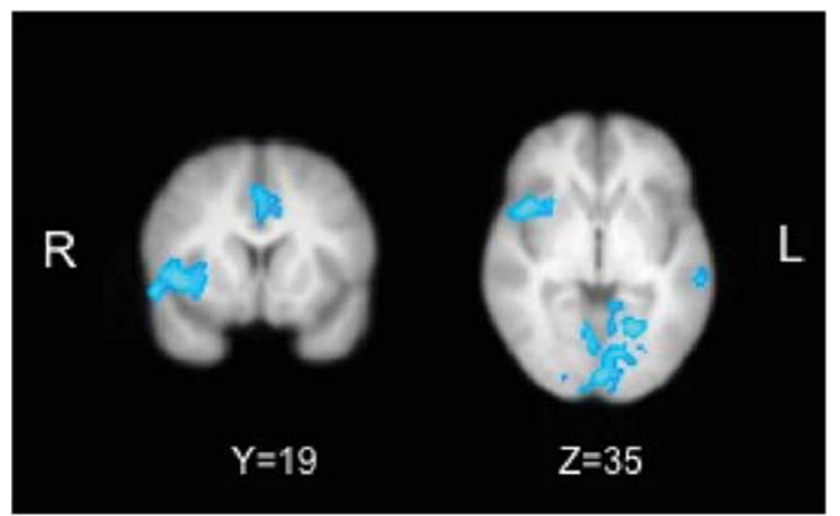

Figure 2 Correlation of stopping time (SSRT) with neural activation. Across all subjects, including nonsmokers and smokers, there was a significant negative correlation between stopping time (SSRT) and neural activation in the IFG, insula, and temporal gyrus, as individuals who were able to stop faster (smaller SSRT) showed greater neural recruitment of these regions. $z=66$ ) in the Successful Stop $>$ Baseline contrast (Figure 3a; Supplementary Table 3), and with left IFG $(x=-52, y=14$, $z=14$ ) in the Successful Stop $>$ Go contrast (Figure 3b; Supplementary Table 3). There were no significant correlations with the Successful Stop $>$ Unsuccessful Stop contrast. Cigarette craving and pack years were not significantly associated with neural activation during response inhibition.

\section{Smoking Behavior and Neural Activation During Responding}

To determine whether associations between smoking variables and neural activity were specific to response inhibition, the same smoking variables that were described above, including HSI and pack years, were subjected to correlation analyses with neural activation during the Go response (Go > Baseline and Go > Successful Stopping contrasts). There were no significant correlations between any of the smoking variables examined and neural activation during the Go response, except a negative correlation between HSI and activity in the supplementary motor cortex ( $x=2, y=-8, z=64)$ in the Go $>$ Baseline contrast (data not shown).

\section{DISCUSSION}

In this study of PFC function in late adolescents, smokers did not differ from nonsmokers on task-related PFC activity during response inhibition; however, an index of smoking behavior and dependence was negatively correlated with neural activation during inhibition. These results suggest that either smoking behavior influences PFC function, or that individuals who have low inhibition-related neural activity a priori show more severe smoking behavior. Although previous studies have shown gender differences in adolescent substance users (Medina et al, 2008; Squeglia et al, 2009), none were observed in this sample.

The major contribution to the literature of this study is its examination of brain function during response inhibition in adolescent smokers. Although working memory and attention in adolescent smokers have been studied before (eg, Jacobsen et al, 2007), previous studies have not examined response inhibition, even though adolescent smokers reportedly have deficits in response inhibition (Dinn et al, 2004). Previous studies have also focused less on examining relationships between smoking and neural activation. Studying this phenomenon in adolescents is of utmost importance, given the high prevalence of initiation

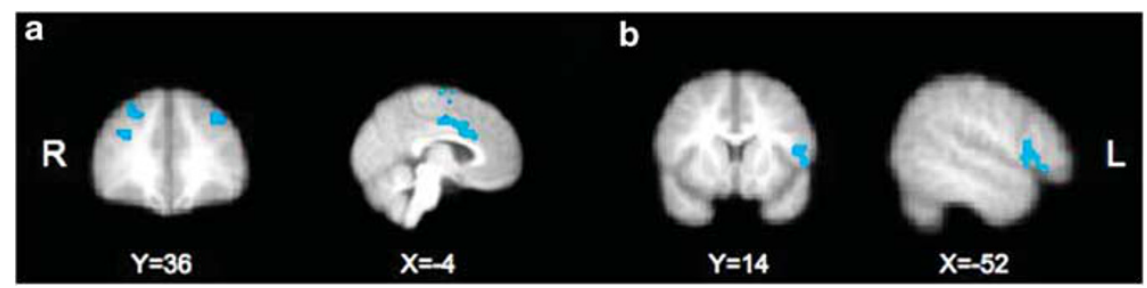

Figure 3 Heaviness of Smoking Index and neural activation. In smokers, HSI was negatively correlated with left and right MFG $(x=-34, y=36, z=38$; $x=30, y=36, z=44)$, cingulate gyrus $(x=0, y=14, z=28)$, supplementary motor cortex $(x=0, y=-2, z=62)$, orbitofrontal cortex $(x=-26, y=56$, $z=2)$, and right superior frontal gyrus $(x=24, y=-2, z=66)$ in the Successful Stop $>$ Baseline contrast (a), and with left IFG $(x=-52, y=14, z=14)$ in the Successful Stop $>$ Go contrast (b). There were no significant correlations with the Successful Stop $>$ Unsuccessful Stop contrast. 
of smoking in adolescents. In fact, individuals who do not start smoking as teenagers are unlikely ever to do so (Sussman, 2002).

The similarity in performance of adolescent smokers and nonsmokers on the SST is consistent with a previous finding that adult smokers do not differ from nonsmokers on SSRT (Monterosso et al, 2005). The lack of a performance deficit on the SST in late adolescent smokers tested here or in adult smokers (Monterosso et al, 2005) suggests that motor response inhibition may be maintained through compensation despite potential effects of smoking on related cognitive processes.

\section{Associations of Smoking Behavior with Brain Function}

Lower levels of neural activation were associated with behavioral features of cigarette smoking. Greater HSI was associated with proportionally less cortical activity in IFG, MFG, SFG, cingulate, and areas of motor cortex during successful response inhibition. The associations observed between smoking behavior and neural activation suggest that adolescents who are heavier smokers show neural profiles that are more similar to adult smokers (eg, atypical neural recruitment compared to nonsmokers) (Azizian et al, 2010; Xu et al, 2007) than adolescents who smoke fewer cigarettes and exhibit less nicotine dependence. To ensure that those participants who were heavier smokers in this sample were not actually adults (19-21 years of age), correlation analyses were conducted, and they revealed no association between age and smoking behavior or cumulative exposure We speculate that, although some effects of smoking on the brain may not be evident soon after smoking initiation in adolescence, they may evolve when smoking persists from adolescence through adulthood (Azizian et al, 2010), or when smoking in adolescence increases to levels seen in adulthood. Notably, although studies of adults show strong right-lateralized activation in the IFG during response inhibition, the findings reported here are left lateralized in IFG and bilateral in other frontal regions, possibly reflecting a greater reliance on a more distributed network in immature (Brown et al, 2005; Durston et al, 2006) and patient (Jacobsen et al, 2007; Tapert et al, 2007) than adult samples.

Smoking behavior in the current sample was similar to previous findings on adolescent smokers (Ernst et al, 2009a). For instance, Ernst et al (2009a) reported an average daily consumption of 2-5 cigarettes/day in a sample of more than 75 adolescents (aged 12-16 years). Our data on cigarette dependence agree with a previous comparison of the CDS-12 between adult (ages 20-74) and adolescent (ages 12-19) smokers $(n=3,009)$, which showed mean values of $44.6(\mathrm{SD}=11.1)$ and $36.9(\mathrm{SD}=9.7)$, respectively (Etter et al, 2003). However, adolescent smokers who smoke more frequently than the subjects studied here (average of 6.7 cigarettes/day) differed from nonsmokers in cognitive and neural measures (eg, Jacobsen et al, 2009), consistent with observations involving adults (Azizian et al, 2010). For example, adolescents who smoked an average of 11.4 cigarettes/day had greater activation than nonsmokers in left ventrolateral PFC and left inferior parietal lobe, and reduced functional connectivity between these regions with increasing memory load (Jacobsen et al, 2007). Aside from differences in recent smoking, duration of smoking abstinence is also potentially important, with 24-h abstinence accounting for the largest reported differences between adolescent smokers and nonsmokers (Jacobsen et al, 2007).

\section{Neurodevelopmental Vulnerability to Nicotine}

There is evidence to suggest that the developing brain is particularly vulnerable to the effects of nicotine (Dwyer et al, 2009). For instance, there are greater nicotine-induced increases in extracellular dopamine in the nucleus accumbens of the adolescent rat than in the adult (Shearman et al, 2008). Adolescent rodents also exhibit greater nicotineinduced gene expression (eg, c-fos) in frontostriatal circuitry than adults (Schochet et al, 2005). These effects parallel the sensitivity of adolescent rodents to the rewarding properties of nicotine, acquiring intravenous nicotine self-administration more readily and taking larger amounts than adults (Chen et al, 2007; Levin et al, 2007). In contrast, conditioned place preference and taste aversion procedures show that high doses of nicotine are perceived as aversive in adult but not in adolescent animals (Shram et al, 2006; Torres et al, 2008). In summary, exposure to nicotine during adolescence may preferentially interfere with frontostriatal circuitry, and these effects may subsequently contribute to poor regulatory behaviors and susceptibility to nicotine dependence (Dwyer et al, 2009).

\section{Neurodevelopment in Adolescent Substance Users}

Numerous studies have explored the neurodevelopment of frontostriatal circuitry in adolescent drug abusers (Jacobus et al, 2009), but generally have not determined whether observed abnormalities reflect risk factors for substance use disorders or neurotoxic effects of the substances. With respect to adolescent smoking, untoward effects may result from neurotoxicity of tobacco smoke and excessive stimulation of nicotinic acetylcholine receptors during development (Dwyer et al, 2009). Structural studies suggest that volumetric abnormalities in the brains of adolescent substance users arise from the neurotoxic effects. For instance, in one study, alcohol users had smaller hippocampal volumes than matched control subjects, even though the groups did not differ with respect to family history of substance use disorders, parental SES, IQ, and other preexisting differences that could presumably affect brain morphology (Medina et al, 2008). Functional studies on adolescents who abuse illegal drugs, (Jacobsen et al, 2007, 2009; Tapert et al, 2007) provided some results similar to those reported here. For instance, our observation that adolescents who had longer histories of tobacco smoking showed less prefrontal engagement resembles the finding that duration of regular marijuana use by adolescents was negatively correlated with activation in BA 10 during response inhibition (Tapert et al, 2007).

\section{Potential Confounds/Limitations}

Chronic smoking decreases basal cerebral blood flow (Kubota et al, 1983) and increases hematocrit (Isaka et al, 1993), which increases the blood oxygenation level-dependent (BOLD) contrast (Levin et al, 2001). As the fMRI signal 
in this study is based on BOLD contrasts, group differences in the coupling between neural function and associated increases in cerebral blood could introduce a potential confound for interpretation of BOLD signal change (Jacobsen et al, 2002). However, BOLD signal did not differ significantly between conditions of nicotine or saline infusion in a study of adult smokers (ages 18-37 years) (Jacobsen et al, 2002), supporting the conclusion that changes in BOLD signal observed during fMRI studies of nicotine effects can be attributed to direct neuronal effects of the drug rather than its effects on the cerebral vasculature (Jacobsen et al, 2002).

The absence of significant group differences in stopping ability, whereas inhibition-related activation in the PFC was correlated negatively with clinical indices of smoking behavior is not completely explained. This dissociation suggests that compensatory mechanisms maintain performance despite effects of smoking on circuitry that supports response inhibition. Absence of group differences in inhibition-associated activation may be a limitation because of sample size, although a sample of 25 participants per group is not small in comparison with most fMRI studies. In this regard, the smoker group had substantial variability in prefrontal cortical function and in indices of smoking behavior. Although this variability was useful in demonstrating associations between smoking behavior and brain activity, it worked against observing a between-group difference, given the sample size.

It is possible that the nonsmokers in this study were particularly abstemious, as reflected in significantly lower levels of alcohol consumption and nonsignificantly lower levels of marijuana use in this group as compared with the smokers. This potential confound may be especially difficult to eliminate in comparisons of smokers with nonsmokers. Nonetheless, the criterion for definition of a nonsmoker in this study $(<5$ cigarettes/lifetime) was no more rigorous than one used in previous studies of adolescents (eg, Jacobsen et al, 2007 ( $<2$ cigarettes/lifetime)). There are known effects of abstinence on brain activity (eg, Azizian et al, 2010; McBride et al, 2006; Mendrek et al, 2006; Rose et al, 2007; Sweet et al, 2010). However, we did not impose abstinence in this sample because we wanted to preclude withdrawal from influencing cognitive performance and neural activation in smokers. The smokers in this study were not heavy smokers, and retrospective reports of nicotine withdrawal over the 7 days before testing (first nine items of the MNWS) indicated that the young smokers tested here generally did not suffer high levels of withdrawal. Nonetheless, there is a slight possibility that not controlling for duration of abstinence potentially confounded the results.

Our study did not determine whether the associations between smoking behaviors and activation related to response inhibition were caused by or preceded the initiation of smoking. It is possible that individuals who have a greater deficit in cortical function a priori have more trouble resisting smoking and ultimately become nicotine dependent. Alternatively, this study might have captured the initial manifestations of the effects of smoking on prefrontal cortical activity, as smokers with longer smoking histories exhibited more aberrant prefrontal function. Answering these questions would require a longitudinal study, involving participants before they initiated smoking behavior.

\section{CONCLUSION}

Indices of smoking behavior were negatively related to PFC function during response inhibition in adolescent smokers. The lack of significant differences in the patterns and levels of neural activation in adolescent smokers and nonsmokers during response inhibition suggests that early interventions during adolescence may prevent the transition from cigarette use to dependence that is prevalent during late adolescence (Curry et al, 2009).

\section{ACKNOWLEDGEMENTS}

The research described in this article was funded, in part, by a grant from Philip Morris USA. Additional funding was provided by an endowment from the Thomas $\mathrm{P}$ and Katherine K Pike Chair in Addiction Studies and a gift from the Marjorie M Greene Trust. None of the sponsors had any involvement with the design, collection, analysis, or interpretation of data, writing the manuscript, or the decision to submit the manuscript for publication.

\section{DICSLOSURE}

Research support was supplied to Dr London by Philip Morris USA under UCLA contract (number 20063287). Philip Morris USA did not have any input on the design of the studies, data analysis, or interpretation. None of the other authors have any conflicts of interest or financial disclosures to report.

Dr Adriana Galván, Principal Investigator, takes responsibility for the integrity of the data and the accuracy of the data analysis. All of the authors had full access to all the data in the study.

\section{REFERENCES}

Aron AR, Poldrack RA (2006). Cortical and subcortical contributions to stop signal response inhibition: role of the subthalamic nucleus. J Neurosci 26: 2424-2433.

Azizian A, Montetosso J, O’Neill J, London ED (2009). Magnetic resonance imaging studies of cigarette smoking. In: Henningfield JE (ed). Nicotine Psychopharmacology Handbook of Experimental Pharmacology. Springer-Verlag: Berlin Heidelberg. pp 113-143.

Azizian A, Nestor LJ, Payer D, Monterosso JR, Brody AL, London ED (2010). Smoking reduces conflict-related anterior cingulate activity in abstinent cigarette smokers performing a Stroop task. Neuropsychopharmacology 35: 775-782.

Beckmann CF, Smith SM (2004). Probabilistic independent component analysis for functional magnetic resonance imaging. IEEE Trans Med Imaging 23: 137-152.

Borland R, Yong HH, O'Connor RJ, Hyland A, Thompson ME (2010). The reliability and predictive validity of the Heaviness of Smoking Index and its two components: findings from the International Tobacco Control Four Country study. Nicotine Tob Res 12(Suppl): S45-S50.

Brown TT, Lugar HM, Coalson RS, Miezin FM, Petersen SE, Schlaggar BL (2005). Developmental changes in human cerebral functional organization for word generation. Cereb Cortex 15: 275-290. 
Center for Disease Control and Prevention (2008). Cigarette smoking among adults - United States, 2006. MMWR Morbidity and Mortality Weekly Report 56: 1157-1161.

Chen H, Matta SG, Sharp BM (2007). Acquisition of nicotine selfadministration in adolescent rats given prolonged access to the drug. Neuropsychopharmacology 32: 700-709.

Chikazoe J, Jimura K, Hirose S, Yamashita K, Miyashita Y, Konishi S (2009). Preparation to inhibit a response complements response inhibition during performance of a stop-signal task. J Neurosci 29: 15870-15877.

Congdon E, Mumford J, Cohen J, Galván A, Aron A, Xue G et al (2010). Engagement of large-scale networks is related to individual differences in inhibitory control. NeuroImage 53: 653-663.

Counett D, Spijker S, Van de Burgwal L, Hogenboom F, Schoffelmeer A, DeVries T et al (2009). Long-lasting cognitive deficits resulting from adolescent nicotine exposure in rats. Neuropsychopharmacology 34: 299-306.

Courvoiser D, Etter J (2010). Comparing the predictive validity of five cigarette dependence questionnaires. Drug Alcohol Depend 107: $128-133$.

Curry SJ, Mermelstein RJ, Sporer AK (2009). Therapy for specific problems: youth tobacco cessation. Annu Rev Psychol 60: 229-255.

Dinn WM, Aycicegi A, Harris CL (2004). Cigarette smoking in a student sample: neurocognitive and clinical correlates. Addict Behav 29: 107-126.

Dorn L, Dahl RE, Woodward H, Biro F (2006). Defining the boundaries of early adolescence: a users' guide to assessing pubertal status and pubertal timing in research with adolescents. App Dev Sci 10: 30-56.

Duann JR, Ide JS, Luo X, Li CSR (2009). Functional connectivity delineates distinct roles of the inferior frontal cortex and presupplementary motor area in stop signal inhibition. J Neurosci 29: 10171-10179.

Durston S, Davidson MC, Tottenham N, Galvan A, Spicer J, Fossella J et al (2006). A shift from diffuse to focal cortical activity with development. Dev Sci 9: 1-8.

Dwyer JB, McQuown SC, Leslie FM (2009). The dynamic effects of nicotine on the developing brain. Pharmacol Ther 122: 125-139.

Ernst M, Luckenbaugh DA, Moolchan ET, Temple VA, Jenness J, Korelitz KE et al. (2009a). Decision-making and facial emotion recognition as predictors of substance-use initiation among adolescents. Addict Behav 35: 286-289.

Ernst M, Romeo RD, Andersen SL (2009b). Neurobiology of the development of motivated behaviors in adolescence: a window into a neural systems model. Pharmacol Biochem Behav 93: 199-211.

Etter J (2008). Comparing the validity of the cigarette dependence scale and the fagerström test for nicotine dependence. Drug Alcohol Depend 95: 152-159.

Etter JF, Le Houezec J, Perneger T (2003). A self-administered questionnaire to measure dependence on cigarettes: the cigarette dependence scale. Neuropsychopharmacology 28: 359-370.

Fagerström KO, Schneider NG (1989). Measuring nicotine dependence: a review of the Fagerström Tolerance Questionnaire. J Behav Med 12: 159-182.

Fountain S, Rowan J, Kelley B, Willey A, Nolley E (2008). Adolescent exposure to nicotine impairs adult serial pattern learning in rats. Exp Brain Res 187: 651-656.

Galván A (2010). Adolescent development of the reward system. Front Hum Neurosci 4: 1-9.

Galván A, Hare TA, Parra CE, Penn J, Voss H, Glover G et al (2006). Earlier development of the accumbens relative to orbitofrontal cortex might underlie risk-taking behavior in adolescents. J Neurosci 26: 6885-6892.

Giedd JN, Rapoport J (2010). Structural MRI of pediatric brain development: What have we learned and where are we going? Neuron 67: 728-734.
Hughes JR, Hatsukami DK (2003). Minnesota Withdrawal Scale. Retrieved from http://www.uvm.edu/ hbpl/minnesota/2005/Behavior \%20Rating\%20Scale\%20-\%20Self\%20Report.pdf (Last accessed 16 August 2010).

Isaka Y, Ashida K, Imaizumi M, Abe H (1993). Effect of chronic smoking on regional cerebral blood flow in asymptomatic individuals. Yakubutsu Seishin Kodo 13: 191-198.

Jacobsen LK, Gore JC, Skudlarski P, Lacadie CM, Jatlow P, Krystal JH (2002). Impact of intravenous nicotine on BOLD signal response to photic stimulation. Magn Reson Imaging 20: $141-145$.

Jacobsen LK, Mencl W, Constable R, Westerveld M, Pugh K (2007). Impact of smoking abstinence on working memory neurocircuitry in adolescent daily tobacco smokers. Psychopharmaco$\log y$ 193: 557-566.

Jacobsen LK, Picciotto M, Heath C, Mencl W, Gelernter J (2009). Allelic variation of calsyntenin 2 (CLSTN2) modulates the impact of developmental tobacco smoke exposure on mnemonic processing in adolescents. Biol Psychiatry 65: 671-679.

Jacobus J, McQueeny T, Bava S, Schwiensburg BC, Frank LR, Yang TT et al (2009). White matter integrity in adolescents with histories of marijuana use and binge drinking. Neurotoxicol Teratol 31: 349-355.

Jarvik ME, Madsen DC, Olmstead RE, Iwamoto-Schaap PN, Elins JL, Benowitz NL (2000). Nicotine blood levels and subjective craving for cigarettes. Pharmacol Biochem Behav 66: 553-558.

Jenkinson M, Bannister P, Brady M, Smith S (2002). Improved optimization for the robust and accurate linear registration and motion correction of brain images. NeuroImage 17: 825-841.

Kubota K, Yamaguchi T, Abe Y, Fujiwara T, Hatazawa J, Matsuzawa T (1983). Effects of smoking on regional cerebral blood flow in neurologically normal subjects. Stroke 14: 720-724.

Levin ED, Lawrence SS, Petro A, Horton K, Rezvani AH, Seidler FJ et al (2007). Adolescent vs adult-onset nicotine self-administration in male rats: duration of effect and differential nicotinic receptor correlates. Neurotoxicol Teratol 29: 458-465.

Levin J, Frederick BB, Ross M, Fox J, von Rosenberg H, Kaufman M et al (2001). Influence of baseline hematocrit and hemodilution on BOLD fMRI activation. Magn Reson Imaging 19: 1055-1062.

Li CS, Huang C, Constable RT, Sinha R (2006). Imaging response inhibition in a stop-signal task: neural correlates independent of signal monitoring and post-response processing. J Neurosci 26: 186-192.

Logan GD (1994). On the ability to inhibit thought and action: a users' guide to the stop-signal paradigm. In: Dagenbach D, Carr TH (eds). Inhibitory Processes in Attention, Memory and Language. Academic Press: San Diego, pp 214-249.

Mathers M, Toumbourou J, Catalano R, Williams J, Patton G (2006). Consequences of youth tobacco use: a review of prospective behavioral studies. Addiction 101: 948-958.

McBride D, Barrett S, Kelly J, Aw A, Dagher A (2006). Effects of expectancy and abstinence on the neural responses to smoking cues in cigarette smokers: an fMRI study. Neuropsychopharmacology 31: 2728-2738.

McNamee RL, Dunfee KL, Luna B, Clark DB, Eddy WF, Tarter RE (2008). Brain activation, response inhibition, and increased risk for substance use disorder. Alcohol Clin Exp Res 32: 405-413.

Medina KL, McQueeny T, Nagel BJ, Hanson KL, Scheweinsburg AD, Tapert SF (2008). Prefrontal cortex volumes in adolescents with alcohol use disorders: unique gender effects. Alcohol Clin Exp Res 32: 386-394.

Mendrek A, Monterosso J, Simon S, Jarvik M, Brody AL, Olmstead R et al (2006). Working memory in cigarette smokers: comparison to non-smokers and effects of abstinence. Addict Behav 31: 833-844.

Monterosso JR, Aron AR, Cordova X, Xu J, London ED (2005). Deficits in response inhibition associated with chronic methamphetamine abuse. Drug Alcohol Depend 79: 273-277. 
Neural correlates

A Galván et al

Rose JE, Behm FM, Salley AN, Bates JE, Coleman RE, Hawk TC et al (2007). Regional brain activity correlates of nicotine dependence. Neuropsychopharmacology 32: 2441-2452.

Schochet T, Kelley A, Landry C (2005). Differential expression of arc mRNA and other plasticity-related genes induced by nicotine in adolescent rat forebrain. Neuroscience 135: 285-297.

Schweinsburg A, Paulus M, Bartlett V, Killeen L, Caldwell L, Pulido C et al. (2004). An fMRI study of response inhibition in youths with a family history of alcoholism. Ann NY Acad Sci 1021: 391-394.

Shearman E, Fallon S, Sershen H, Lajtha A (2008). Nicotineinduced monoamine neurotransmitter changes in the brain of young rats. Brain Res Bull 76: 626-639.

Shram MJ, Funk D, Li Z, Lê AD (2006). Periadolescent and adult rats respond differently in tests measuring the rewarding and aversive effects of nicotine. Psychopharmacology (Berl) 186: 201-208.

Somerville LH, Casey B (2010). Developmental neurobiology of cognitive control and motivational systems. Curr Opin Neurobiol 20: $236-241$.

Squeglia LM, Jacobus J, Tapert SF (2009). The influence of substance use on adolescent brain development. Clin EEG Neurosci 40: 31-38.

Sweet LH, Mulligan RC, Finnerty CE, Jerskey BA, David SP, Cohen RA et al (2010). Effects of nicotine withdrawal on verbal working memory and associated brain response. Psychiatry Res 183: 69-74.
Sussman S (2002). Effects of sixty-six adolescent tobacco use cessation trials and seventeen prospective studies of self initiated quitting. Tob Induc Dis 1: 35-81.

Tapert S, Schweinsburg A, Drummond S, Paulus M, Brown S, Yang $\mathrm{T}$ et al (2007). Functional MRI of inhibitory processing in abstinent adolescent marijuana users. Psychopharmacology (Berl) 194: 173 - 83 .

Torres OV, Tejeda HA, Natividad LA, O'Dell LE. (2008). Enhanced vulnerability to the rewarding effects of nicotine during the adolescent period of development. Pharmacol Biochem Behav 90: 658-663.

Velanova K, Wheeler ME, Luna B (2008). Maturational changes in anterior cingulated and frontoparietal recruitment support the development of error processing and inhibitory control. Cereb Cortex 18: 2505-2522.

Woolrich M (2008). Robust group analysis using outlier inference. NeuroImage 41: 286-301.

Xu J, Mendrek A, Cohen M, Monterosso J, Rodriguez P, Simon S et al (2005). Brain activity in cigarette smokers performing a working memory task: effect of smoking abstinence. Biol Psychiatry 58: 143-150.

Xu J, Mendrek A, Cohen M, Monterosso J, Simon S, Jarvik M et al (2007). Effect of cigarette smoking on prefrontal cortical function in nondeprived smokers performing the Stroop Task. Neuropsychopharmacology 32: 1421-1428.

Supplementary Information accompanies the paper on the Neuropsychopharmacology website (http://www.nature.com/npp) 